\title{
Latent energy savings due to the innovative use of advisory speeds to avoid occupation conflicts
}

\author{
F. Mehta, C. Rößiger \& M. Montigel \\ systransis Ltd., Switzerland
}

\begin{abstract}
Track occupation conflicts are frequent in standard railway operations today. Train drivers, who are not aware of such conflicts in advance, are forced to stop, which results in additional delays, timetable instability, and a waste of energy. This could be avoided if they were informed about the conflict and had a chance to adapt their driving behaviour accordingly. The innovative computer-based train control system "Automatic Functions Lötschberg" (AF), developed by systransis Ltd, tries to reduce these negative effects by sending advisory speeds to the drivers of conflict affected trains in the Lötschberg base tunnel.

This article presents the results of a study done using real operational data from the Lötschberg base tunnel to estimate the energy savings due to the AF sending advisory speeds. These results are then extrapolated to estimate the latent energy savings that could be achieved if a system like the AF were in operation over the entire Swiss railway network.
\end{abstract}

Keywords: advanced train control, energy savings, advisory speeds.

\section{Introduction}

With a length of $34.6 \mathrm{~km}$ under the Swiss Alps, the Lötschberg base tunnel is currently the longest land tunnel in the world. The computer-based train control system "Automatic Functions Lötschberg" (AF), developed by systransis Ltd. as a subcontractor of Thales Ltd, has been monitoring and controlling the train traffic through the Lötschberg base tunnel since its opening in December 2007.

The topology of the tunnel introduces special challenges in its operation. The northernmost two thirds of the tunnel is a single-track section, which feeds into a two-track section in the south via the high-speed point "W60". Figure 1 illustrates this topology. Solving track occupation conflicts between trains is especially important since the single-track section needs to be used optimally. 


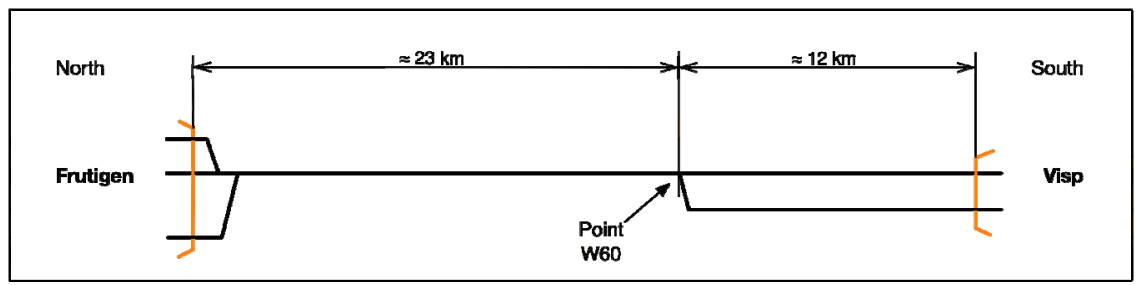

Figure 1: Topology of the Lötschberg base tunnel.

The tunnel is equipped with ETCS Level 2 as its train protection system which allows continuous tracking of train speeds and positions, and communication to the on board units of each train.

One essential function of the AF is forecasting and solving track occupation conflicts by calculating an optimal speed trajectory for a train affected by a conflict that would otherwise have to stop or slow down. It then sends advisory speeds via GSM-R to the train driver who uses them as a recommendation for his onward journey. This gives him the possibility to solve the conflict by preemptively slowing down, instead of eventually being forced to stop. Advisory speeds are sent to the affected train as text messages in regular time intervals of 30 seconds. A final message "vopt $=$ vmax" is sent when the advisory speed limit is to be lifted.

The primary goal of sending advisory speeds to train drivers is to reduce collateral delays and minimise train stops caused by conflicts, and thereby maintain capacity and timetable stability. Figure 2 illustrates the approach of solving track occupation conflicts used by the AF. More details on the computational aspects and use of the AF in the Lötschberg base tunnel can be found in Montigel et al. [1] and Montigel [2].

Although not its main aim, a welcome side effect of this optimisation is the reduction of the traction energy needed by trains to travel through the tunnel. This claim is intuitive: energy consumption should be lower if a train is not required to come to a full stop. In order to test this claim empirically, the following study was undertaken to quantitatively estimate how much traction energy was saved in this way using real operational data.

Technologies for increasing energy efficiency in the context of railway operation are receiving increased attention. A review of these technologies can be found in [7]. Increasing energy efficiency through energy-optimal train trajectories have also been studied extensively. The possibilities for computing and using such trajectories are described in detail in Albrecht [8], Howlett and Pudney [9], and Franke et al. [10]. Lüthi [4] discusses the energy saved as a result of integrated real-time rescheduling. Mitchell [11] discusses the impact of advisory systems on energy savings and uses a simulation-based model to quantitatively estimate these savings. The novelty of the work described in this article is that this is the first time that data from real-world commercial railway operations with traffic flow optimisation is available and used to determine energy savings. 


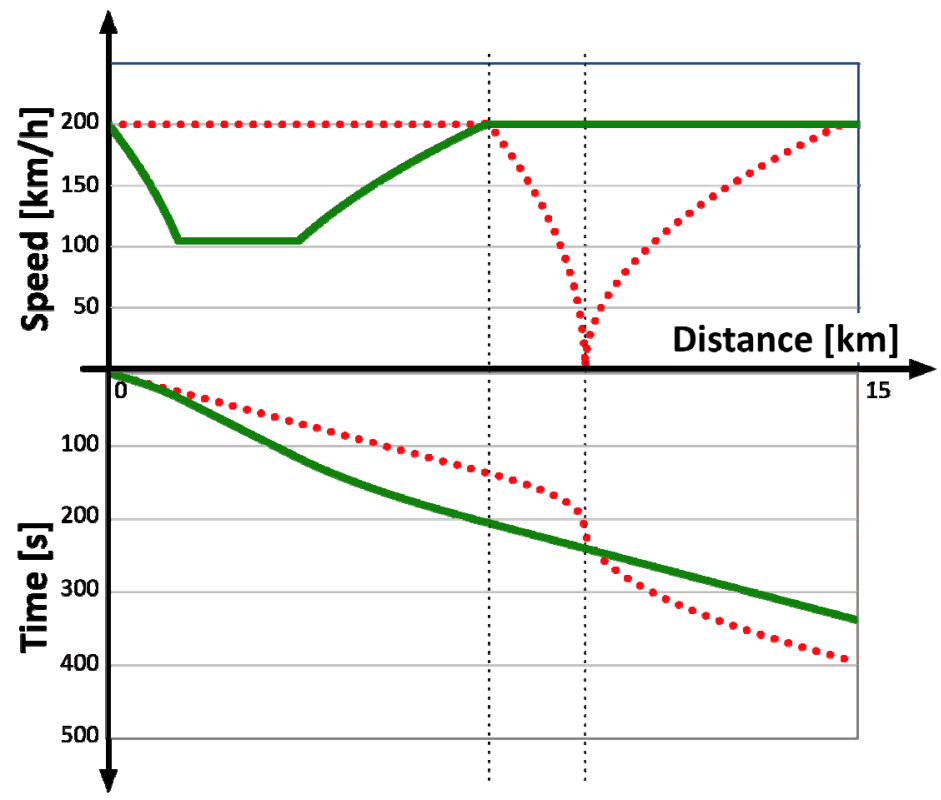

Figure 2: $\quad$ Time gained by sending advisory speed. The solid line is optimised and avoids a halt.

\section{Methodology used}

The following general methodology was used to estimate the energy savings as a result of sending advisory speeds. Pre-recorded operational data (i.e. log files generated by the AF) from the tunnel was used to extract all train movements in the tunnel during which an advisory speed was sent to resolve an occupation conflict. This operational data was used to reconstruct the actual train trajectory through the tunnel, and thereby the actual traction energy consumed for each such train run was calculated.

In order to compute the energy savings, a comparison of the actual traction energy consumed with the energy that would have been consumed if no advisory speeds were sent (i.e. for the non-optimised case) needs to be done. The functionality of the AF to send advisory speeds has been continuously active since the start of operation of the tunnel. Since such a study cannot warrant turning off this functionality just for test purposes it was not possible to directly measure the energy consumed if no advisory speeds were sent. Therefore, assumptions about the behaviour of train drivers for the non-optimised case needed to be made. Based on these assumptions, a non-optimised train trajectory was generated and used to calculate the energy consumption for the nonoptimised case. This was then used to estimate the energy saved as a result of sending advisory speeds. 


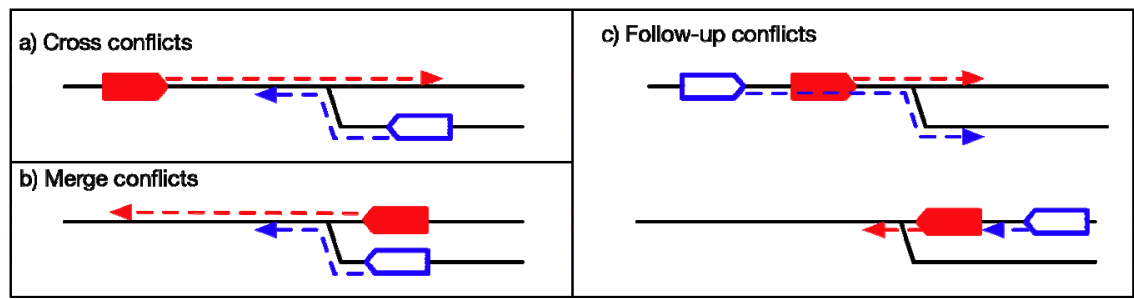

Figure 3: Conflict types for which an advisory speed is sent in the tunnel.

The rest of this section describes the above methodology in greater detail.

\subsection{Conflicts considered in this study}

The AF sends advisory speeds to train drivers in the tunnel in the following three general cases:

a. Cross conflicts

b. Merge conflicts

c. Follow-up conflicts

Figure 3 illustrates these three conflict types. The filled train is the one causing the conflict, and the hollowed train is the one affected by it. The hollowed train receives advisory speeds. It should be noted that the AF also solves other types of conflicts in the tunnel, but these are disregarded in this study.

Out of these three cases, cross and merge conflicts are of interest as far as energy savings are concerned since they will, in most cases, result in a full halt of the affected (hollowed) train if not solved by the AF optimisations. Follow-up conflicts are not currently considered, as this would significantly complicate the method used, requiring more than one train to be considered per conflict case. Furthermore, such follow-up conflicts are rare in practice since the train dispatcher takes care to avoid them by positioning faster trains before slower ones.

Another point to note is that conflicts are often interdependent. Solving a conflict favourably in the present time can avoid future conflicts. This study though only considers individual conflicts in order to avoid too much speculation into the future.

\subsection{Area of interest}

The AF log files are processed to extract the following data for each train affected by a cross or merge conflict in the tunnel:

- Train characteristics: engines, weight, class (passenger or freight)

- Position and speed reports at various times

- Transmitted advisory speeds at various times

The analysis is restricted to the section of the travelled train path affected by the receipt of advisory speeds. The start position of this area of interest is the 


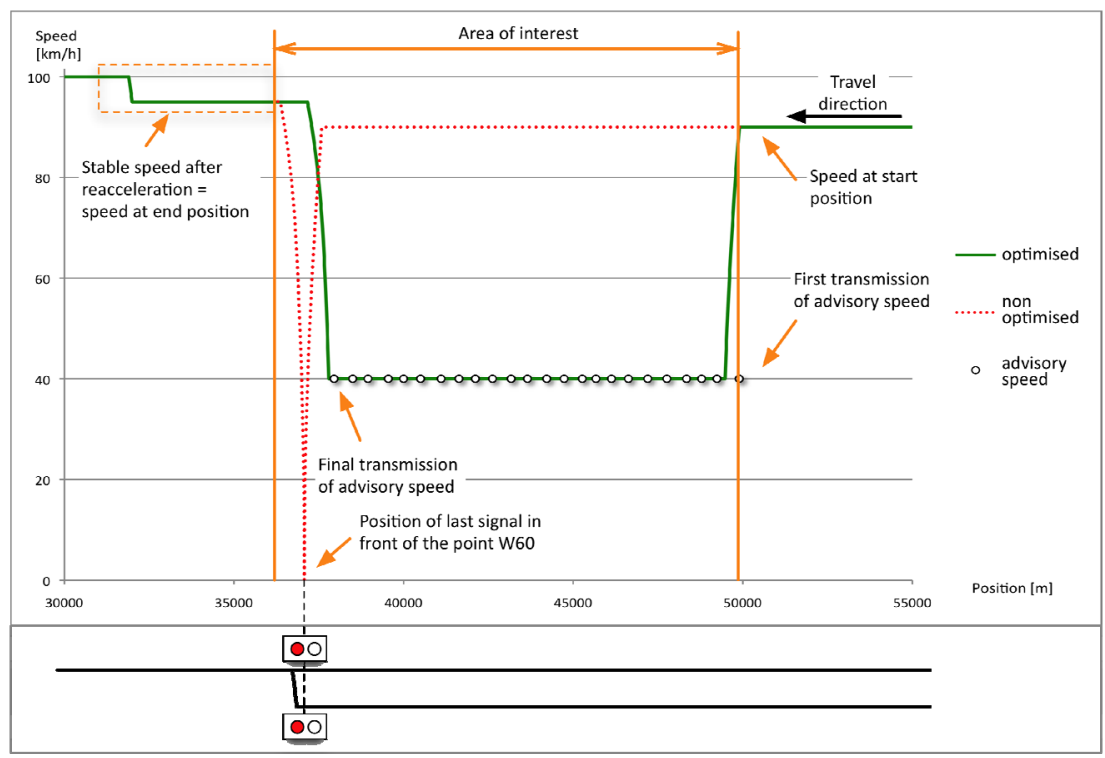

Figure 4: Area of interest and train trajectories for the optimised and non-optimised cases.

position where the affected train receives the first advisory speed. The end position of this area of interest is the position where the train has finished its reacceleration after the merging point $\mathrm{W} 60$ and reaches a stable speed over a defined distance.

Figure 4 illustrates how this area of interest is determined using the optimised (actual) train trajectory, and how the non-optimised (estimated) trajectory is calculated using the start and end speeds and positions, and the position of the last signal in front of the point W60. The calculation of the nonoptimised trajectory will be described in detail later. Note that the figure is to be read from right to left since the affected trains travel in the direction of decreasing mileage.

\subsection{Energy calculation model}

Energy consumption is calculated using the following standard formula, by numerically integrating the traction force exerted by the engines $\left(F_{i}\right)$ over the respective travelled distances $\left(s_{i}\right)$ :

$$
E=\sum_{i=1}^{n} F_{i} s_{i}
$$

The following components of the traction force are considered:

- Rolling resistance (dependent on speed, always positive)

- Tunnel resistance (dependent on speed, always positive) 
- Gradient resistance (positive for uphill tracks, negative for downhill)

- Free acceleration force (positive for acceleration, negative for braking)

The free acceleration force is determined using the position and speed reports extracted from the AF log files. The resistances are assumed to be constants that depend on the actual speed and train class. The sum of all these forces needs to be applied by the engines and is used to calculate the traction energy needed by them.

\subsection{Regenerative braking}

It is assumed that a certain ratio of the negative free acceleration force could be recuperated. This ratio accounts for the efficiency of the regeneration process, the conductive losses of the overhead wire, and the fact that the recuperated energy can only be effectively used if there is an energy consumer currently connected to an interconnected overhead wire. Two values are used for this ratio:

1. For the optimised case it is expected that a high ratio (i.e. $40 \%$ ) of the deceleration energy can be recuperated. This is because the AF calculates advisory speeds in such a way that the required deceleration can be achieved solely using regenerative braking.

2. For the non-optimised case a lower ratio (i.e. 20\%) is assumed because a larger amount of the total braking force has to be provided using mechanical brakes.

\subsection{Estimating the non-optimised case}

For the non-optimised case it is assumed that no advisory speeds are transmitted. The train driver doesn't know about the conflict until he has to brake because of the last signal in front of the point W60.

The calculation of the train trajectory for the non-optimised case uses the start and end speeds and positions, as well as the position of the last signal in front of the point W60 as described earlier. The trajectory consists of the following phases:

1. Travelling with the start speed until hitting the braking curve of the last signal in front of the point

2. Braking to standstill at the signal in front of the point

3. Accelerating to the end speed

The non-optimised curve in Figure 4 illustrates this trajectory. The model used for calculating the non-optimised trajectory is based on the standard train dynamics model contained in Hürlimann [3], which is also the one used in the AF.

Brake applications are modelled as constant decelerations, dependent on the class of the train. Coasting (i.e. speed decrease without application of traction force) was not considered.

For acceleration, the traction capabilities of individual engines are considered. For each engine type, the traction forces dependent on the current speed are used. The free acceleration is calculated using the train weight, dynamic mass factor, class, and the driving resistances described earlier. 
The resulting free acceleration is then used to calculate the traction energy needed to complete the non-optimised trajectory and thereby estimate the energy saved as a result of sending advisory speeds.

\section{Results and discussion}

For this study 746 optimised train journeys over a three-month period from August 2008 to October 2008 were considered. Of these, 117 journeys involved passenger trains, and 629 involved freight trains.

\subsection{Quantitative results}

The total estimated energy savings for these journeys was calculated to be $45,655 \mathrm{kWh}$. The average saved traction energy per optimised journey was therefore $61.2 \mathrm{kWh}$.

Although the absolute energy savings in $\mathrm{kWh}$ varies strongly for each train run, the percentage of the energy saved (with respect to the energy used to cover the area of interest in the non-optimised case) does not vary as significantly. This can be seen in Figure 5. The percentage of total energy saved to cover the area of interest was calculated to be $\mathbf{1 2 . 4 \%}$.

These estimates are still on the conservative side since, as mentioned before, this study though considers individual conflicts as isolated, and does not take into account that present conflicts, if not solved favourably, can lead to future

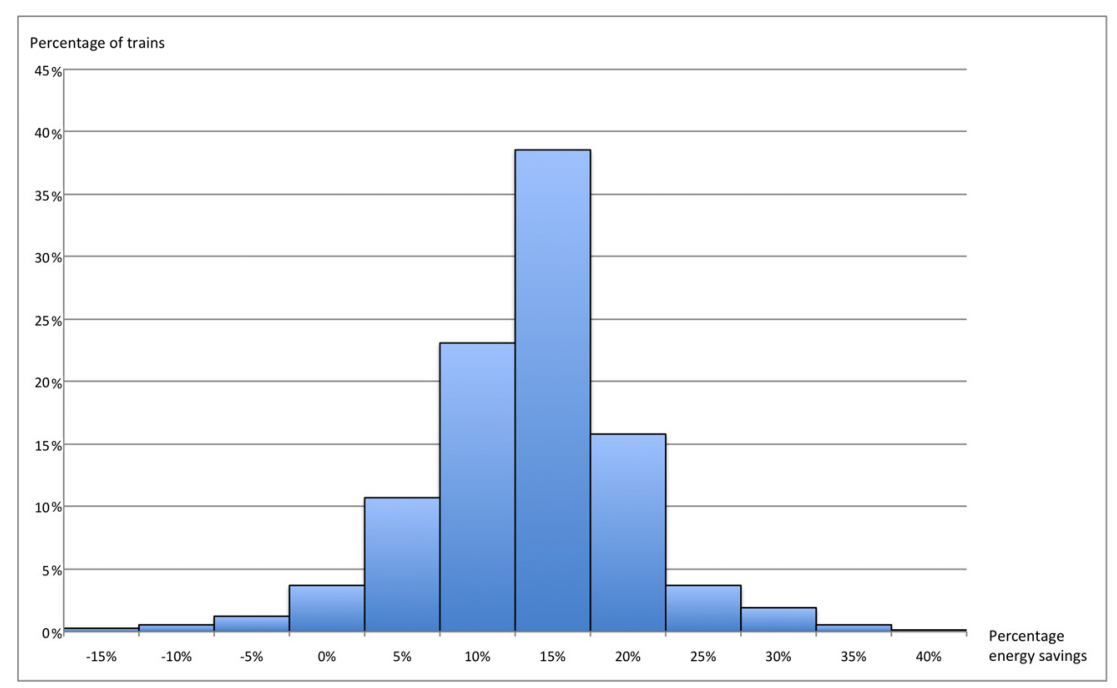

Figure 5: Distribution of the percentage energy saved due to sending advisory speeds. 
conflicts. More conflicts would therefore be observed if advisory speeds were not transmitted, leading to a greater energy consumption in practice for the nonoptimised operation than the estimate calculated above.

What is worth mentioning again is that energy savings are not directly taken into account in the calculation of advisory speeds. They are purely a side effect of reducing the delays resulting from a conflict.

\subsection{Qualitative results}

Apart from the above quantitative analysis, the data was also qualitatively analysed in order to learn more about factors on which energy savings may depend:

\subsubsection{Journey attributes}

It could be thought that the percentage energy savings depends on the train weight, or the ratio between the initial and advisory speeds. However, the analysis of this data did not reveal any such dependencies.

\subsubsection{Train class}

An interesting question is whether energy savings due to freight and passenger trains differ. Passenger trains travel with higher speeds $(160-200 \mathrm{~km} / \mathrm{h})$ through the tunnel and have to spend a higher ratio of their energy to overcome resistive forces. Freight trains are heavier and therefore need longer distances to accelerate. The energy consumption was partitioned for the two train classes with the result that the percentage of saved energy for freight trains is slightly higher $(12.9 \%)$ than that for passenger trains $(10.3 \%)$.

\subsubsection{Regenerative braking}

The impact of regenerative braking on the consumed energy is often emphasized. Since the total amount of recoverable energy is subject to many influences, it is not easy to draw a final conclusion concerning the effectiveness of regenerative braking. The assumption in this study was that all considered trains are capable of regenerative braking and an average ratio of $20 \%$ of the free braking energy could be recuperated in the non-optimised case, and $40 \%$ in the optimised case. For the sake of comparison, energy savings with different ratios $(0 \%-0 \% ; 50 \%$ $100 \%$ ) was calculated. The results for these ratios deviated not more than $1.5 \%$ from the original result. Reasons for this small impact of regenerative braking are:

1. A high ratio of the braking energy is used to overcome resistance forces, which are higher in a tunnel when compared to an open track, leaving only a minor part for recuperation.

2. The distances where regenerative braking can be applied are short compared to the total length of the area of interest.

Eventually, regenerative braking seems not to have a big influence on the energy savings provided by the AF. Nevertheless, it may play a relevant role when the total consumed energy is considered. 


\subsubsection{Driver behaviour}

For this study, operational data from the tunnel was used to compute the energy consumption in the actual case, regardless of whether the train driver obeyed the advisory speeds or not. It would be interesting to see how much the saved energy estimated from the actual data deviates from the optimised trajectory calculated by the AF. To do this an ideally optimised trajectory based on the first advisory speed transmitted by the AF was computed. The resulting estimated energy savings were observed to be $10.1 \%$ compared to the $12.4 \%$ estimated energy savings using the actual travelled trajectory, i.e. not all train drivers obey the advisory speeds strictly and nonetheless consume less energy than estimated by the AF. An explanation for this maybe unexpected result can be that in reality, the train drivers partly applies coasting, which is not considered by the calculations for the actual optimised case. Another point to note is that the aim of sending advisory speeds is to minimise delays, and energy savings is only a side effect of this. The computed optimal trajectory need not therefore consume the least energy. The possibility that the train driver chooses a trajectory that is energetically better is therefore possible.

\section{Latent energy savings for the entire Swiss railway network}

The study described till now estimated that the AF sending advisory speeds saved about $60 \mathrm{kWh}$ of traction energy per conflict. Using this figure, it would be interesting to make a rough extrapolation of the total energy that could be saved, given that a system, such as the AF, were in operation over the entire Swiss railway network.

The following assumptions on the daily train traffic from [5] and [6] are used:

1. About 1500 passenger journeys (only long-distance trains) occur each day, each experiencing on average 2 conflicts leading to an unplanned stop

2. About 2000 freight journeys occur each day, each experiencing on average 3 conflicts leading to an unplanned stop.

It is easy to see that the resulting latent energy savings for an entire year is in the order of $200 \mathrm{GWh}$ (i.e. 200 million $\mathrm{kWh}$ ). The current market value of electrical energy is around CHF 0.20 per $\mathrm{kWh}$. Hence, this translates into a monetary saving of about CHF 40 million in energy costs alone, not to mention the added value and monetary gain due to fewer delays and better timetable stability, and reduced maintenance costs due to less wear and tear.

Since engine efficiency and conductive resistance of the overhead wire have not been taken into account for these figures, the metered energy saved would therefore, in practice, be more than the estimates just calculated. As mentioned before, it also has to be taken into account that the optimum for energy savings does not necessarily have to match the optimum for operational purposes.

\section{Conclusion}

It can be concluded from this study that there exists a significant potential to save energy in railway operations by introducing a computer-based train control 
system like the AF that uses advisory speeds to resolve occupational conflicts in a railway network.

In the case of the Lötschberg base tunnel, as per conservative estimates, it is observed that about $\mathbf{6 0} \mathbf{~ k W h}$ of traction energy is saved per conflict due to advisory speeds. This translates to a savings of $\mathbf{1 2 . 4 \%}$ of the traction energy needed to travel through the conflict-affected region, when compared to an estimated non-optimised train trajectory. It is also observed that this percentage of saved energy does not depend significantly on regenerative braking, conflict size, or class of train.

Even a rough extrapolation of these results for the entire Swiss railway network yields a significant energy savings potential of about 200 GWh for a year. The resulting monetary savings of about CHF $\mathbf{4 0}$ million per year could be therefore well invested in a computer-based train control system like the AF on a nationwide level, which would also provide further benefits such as reduced delays and better timetable stability.

\section{References}

[1] Montigel M., Kleiner C. \& Achermann E., Experience with the Speed and Traffic Optimisation employed in the novel Train Traffic Control Center of the Lötschberg Base Tunnel in Switzerland, Proceedings of Railway Capacity - The Engineering Challenge, 2007.

[2] Montigel M., Operations control system in the Lötschberg Base Tunnel, RTR - European Rail Technology Review 02/2009, 2009.

[3] Hürlimann D., Objektorientierte Modellierung von Infrastrukturelementen und Betriebsvorgängen im Eisenbahnwesen, Diss. ETH Nr. 14281, ETH Zürich, 2001.

[4] Lüthi M., Evaluation of energy saving strategies in heavily used rail networks by implementing an integrated real-time rescheduling system, Comprail 2008 Proceedings, 2008.

[5] Information from Media centre SBB, 2009.

[6] http://www.reisezuege.ch/, queried 24th November 2009 for timetable period 2009/10.

[7] http://www.railway-energy.org, Website for Energy Efficiency Technologies for Railways.

[8] Albrecht, T. Energy-Efficient Train Operation, Chapter in Railway Timetable and Traffic, pp 83-106, Eurail Press, 2008.

[9] Howlett, P.G., Pudney, P.J. Energy-efficient train control, Springer, Berlin, 1995.

[10] Franke, R., Meyer, M., Terwiesch, P. Optimal Control of the Driving of Trains, Automatisierungstechnik 50(12), pp 606-613, 2002.

[11] Mitchell, I, The Sustainable Railway - Use of Advisory Systems for Energy Savings, IRSE Technical Paper, 2009. 